\title{
The epidemiology of invasive pneumococcal disease in older adults in the post-PCV era. Has there been a herd effect?
}

\author{
M. CORCORAN ${ }^{1}$, I. VICKERS ${ }^{1}$, J. MERECKIENE ${ }^{2}$, S. MURCHAN $^{2}$, \\ S. COTTER ${ }^{2}$, M. FitzGERALD ${ }^{2}$, M. MCELLiGOTT ${ }^{1,3}$, M. CAFFERKEY $^{1,3,4}$, \\ D. O'FLANAGAN ${ }^{2}$, R. CUNNEY ${ }^{1,2,4}$ AND H. HUMPHREYS ${ }^{1,3,5}$ \\ ${ }^{1}$ The Irish Pneumococcal Reference Laboratory, Irish Meningitis and Sepsis Reference Laboratory, Temple \\ Street Children's University Hospital, Temple Street, Dublin 1, Ireland \\ ${ }^{2}$ Health Protection Surveillance Centre, Dublin, Gardiner Street, Dublin 1, Ireland \\ ${ }^{3}$ Department of Clinical Microbiology, The Royal College of Surgeons in Ireland, RCSI Education \& Research \\ Centre, Beaumont Hospital, Beaumont, Dublin 9, Ireland \\ ${ }^{4}$ Department of Microbiology, Temple Street Children's University Hospital, Dublin 1, Ireland \\ ${ }^{5}$ Department of Microbiology, Beaumont Hospital, Beaumont, Dublin 9, Ireland
}

Received 30 January 2017; Final revision 17 May 2017; Accepted 19 May 2017; first published online 15 June 2017

\section{SUMMARY}

The 7 and 13-valent pneumococcal conjugate vaccines (PCVs) have reduced the incidence of invasive pneumococcal disease (IPD) in children in many countries. The objective of this work was to assess the impact of PCVs and potential herd-protection in older adults in Ireland. IPD notification and typing data from adults $\geqslant 65$ years of age from July 2007 to June 2016 was assessed using national surveillance data. There was a 94\% reduction in PCV7 serotypes from 2007-2008 to 2015-2016, incidence rate ratio (IRR 0.05, $P<0 \cdot 0001$ ). However, there was no decline in the additional PCV13 (PCV13-7) serotypes over the same period (IRR 0.90) nor in comparison with the pre-PCV13 period 2009-2010 (IRR 0.92). The incidence of serotypes in the 23-valent pneumococcal polysaccharide vaccine only (PPV23-PCV13) and non-vaccine types (NVTs) increased significantly (IRR 2.17, $P=0 \cdot 0002$ and IRR 3.43, $P=0 \cdot 0001$ respectively). Consequently, the overall IPD incidence rate in adults has remained relatively unchanged (from $28 \cdot 66 / 100000$ to $28 \cdot 88 / 100000$, IRR $1 \cdot 01, P=0 \cdot 9477$ ). Serotype 19 A and NVTs were mainly responsible for penicillin resistance in recent years. The decline of PCV7 serotypes indicate that the introduction of PCV7 resulted in herd-protection for adults. However, increases in PPV23PCV13 and NVTs suggest that changes in vaccination strategy amongst older adults are needed to build on the success of PCVs in children.

Key words: Pneumococcal infection, Streptococcus pneumoniae (pneumococcus), Surveillance, typing, vaccine preventable diseases, IPD in adults.

\section{INTRODUCTION}

Streptococcus pneumoniae is a gram-positive bacterial organism that readily colonises the nasopharynx

\footnotetext{
* Author for correspondence: M. Corcoran, The Irish Pneumococcal Reference Laboratory, Irish Meningitis and Sepsis Reference Laboratory, Temple Street Children's University Hospital, Temple Street, Dublin 1, Ireland.

(Email: marytcorcoran@gmail.com, mary.corcoran@cuh.ie)
}

asymptomatically. The organism can cause noninvasive and invasive pneumococcal disease (IPD) such as bloodstream infection/septicaemia, meningitis and invasive pneumonia. IPD remains a considerable cause of morbidity and mortality in both resourcerich and resource-poor countries with the highest disease risk associated with young children and older adults [1]. 
The 7-valent pneumococcal conjugate vaccine (PCV) was developed to provide protection against predominant serotypes associated with paediatric invasive disease at the time of vaccine development [2]. Subsequently, 10-valent and 13-valent vaccines (PCV10/PCV13) were introduced to provide protection against additional serotypes and to address serotype replacement [3-5]. In many countries, the introduction of PCVs has resulted in a significant reduction of IPD in children and also contributed to herd-protection for unvaccinated adults [3, 6-12].

However, vaccination schedules vary according to regions and countries [13-15]. IPD incidence rates and the predominant serotypes may also vary due to patient demographics and vaccination uptake $[3,16-18]$. As a result, it is important that countries monitor the serotype distribution and incidence rates in order to detect and report regional differences and monitor the effect of vaccination.

PCV7 was introduced to the Irish childhood immunisation schedule for children aged 2, 6 and 12 months of age $(2+1$ campaign $)$ in September 2008, with a catch-up campaign for children $<2$ years of age. The PCV13 replaced PCV7 in December 2010 with the vaccine offered to children aged 2, 6 and 12-13 months of age, without the catch up campaign. Uptake of three doses of PCV by 24 months of age has ranged between 90 and $92 \%$ since 2011 [19]. The latest figures indicate that the number of PCV7 and PCV13-7 cases have fallen by 100 and $45 \%$, respectively, in patients $<5$ years of age [20] and is similar to what is reported elsewhere with a $2+1$ schedule $[6-8,10,21]$.

In Ireland adults $\geqslant 65$ years of age are recommended to receive the 23-valent pneumococcal polysaccharide vaccine. As of August 2015, PCV13 is recommended for high-risk adults such as those with immunosuppressive conditions or other riskassociated co-morbidities [22]. However, the uptake of PPV23 remains low among the adult population in Ireland [23]. The most recent figures indicate that between 27 and $36 \%$ of adults $\geqslant 65$ years of age received PPV23 between 2005 and 2013 [23] in comparison with other countries such as Germany $(51 \%)$ [24], the USA (60\%) [15] and the UK (70\%) [25]. However, despite successful PPV23 vaccination campaigns in countries such as the UK, there has been no clear evidence of a reduction of PPV23-PCV13 serotypes among adults. Whereas the addition of PCV13 to the childhood immunisation schedule has reduced the incidence of PCV13 serotypes and the overall incidence of IPD in the adult population $[6,26]$.
The large scale CAPiTA trial conducted in The Netherlands provided PCV13 to over 40000 participant $\geqslant 65$ years of age [27]. The results indicated that the number of invasive infections caused by PCV13 serotypes was lower after vaccination, with efficacy rates of 76 and $49 \%$ for PCV13 and all serotypes, respectively [27]. Further studies have reported no evidence of waning immunity with PCV13 after 5 years [28] unlike with PPV23 [29, 30]. Recent studies in the USA have indicated that the provision of PCV13 followed by a subsequent dose of PPV23 to patients $\geqslant 70$ years of age elicits a higher antibody response to PCV13 serotypes than PPV23 alone [31]. Following this, the USA has introduced one dose of PCV13 and then subsequently immunised with PPV23 to further reduce the burden of IPD in adults [32].

Given the complexity of the evolving predominant serotypes and differences in disease incidence rates, national surveillance of IPD is vital to assess changes that may affect future vaccination policies for different age groups, including the elderly. Here we evaluate the indirect effects of PCVs on adults $\geqslant 65$ years of age in Ireland and discuss the implications.

\section{MATERIALS AND METHODS}

\section{Data collection}

For notification purposes, confirmed IPD cases includes isolation of $S$. pneumoniae by culture or the detection of $S$. pneumoniae nucleic acid or antigens from a normally sterile site [33]. Clinicians and laboratories are legally obliged to notify the Department of Public Health (DPH) of IPD infections in Ireland since 2004. Statutory notification data are collated using the Computerised Infectious Diseases Reporting (CIDR) system, a secure web-based system for collecting and collating data on notifiable infectious diseases in Ireland. Public and private clinical laboratories are requested to refer all culture positive IPD isolates to the Irish Pneumococcal Reference Laboratory (IPRL) for typing. Despite the introduction of molecular technology as an additional method for detection of pathogens, the use of the routine culture techniques remains the core method for detecting culture positive $S$. pneumoniae from sterile samples such as blood and CSF samples. The serotype results are linked with the individual notification events reported each quarter using the CIDR system. Missing or incomplete typing data are continually monitored in each region through the DPH and at a national level 
at the Health Protection Surveillance Centre, each quarter. Laboratories with missing or incomplete typing data are then invited to refer any available isolates to the IPRL for typing. The results of a recent retrospective audit of all national laboratories indicated that all laboratories who reported identifying $S$. pneumoniae from sterile sites were also reporting the findings to DPH, and based on the isolate records there was a high rate of completeness of typing during this period. This paper focuses on confirmed IPD notifications only from culture positive cases in adults $\geqslant 65$ years of age with available serotype data. IPD cases that were confirmed by PCR alone were not included.

\section{Laboratory analysis}

Serotyping was performed on all invasive pneumococcal isolates available by serological reactions using the serum samples from the Staten Serum Institute (DK-2300 Copenhagen S, Denmark) and a multiplex-PCR as previously described [34]. Antimicrobial susceptibility was assessed using the $E$-test method, and results interpreted using the Clinical Laboratory Standards Institute (CLSI) meningitis breakpoints [35]. Resistance to penicillin and cefotaxime are defined as a Minimum Inhibitory Concentration of $\geqslant 0 \cdot 12 \mathrm{mg} / \mathrm{L}$ and $\mathrm{MIC} \geqslant 2 \mathrm{mg} / \mathrm{L}$.

\section{Data analysis}

Incidence rates (IR) were calculated based on the annual population estimates provided by the Central Statistics Office (http://www.cso.ie) and expressed as the number of serotyped isolates from cases per 100000 population $(/ 100000)$. The population estimates for those $\geqslant 65$ years of age in Ireland has increased from 471021 people to 606011 people ( $29 \%$ increase) over the 9 year period. All changes in incidence rates are stratified to take this into account. The isolates were also categorised according to vaccine-associated serotypes: PCV7 serotypes $(4,6 \mathrm{~B}$, $9 \mathrm{~V}, 14,18 \mathrm{C}, 19 \mathrm{~F}, 23 \mathrm{~F})$; the additional serotypes included in PCV13 but not in PCV7 i.e. PCV13-7 serotypes (1, 3, 5, 6A, 7F, 19A); the PPV23 serotypes not covered in the either of the $\mathrm{PCV}$ vaccines i.e. PPV23-PCV13 (2, 8, 9N, 10A, 11A, 12F, 15B, 17F, 20, 22F, 33F); NVT serotypes, non-vaccine associated serotypes and non-typable isolates. The distribution of typed IPD cases among older adults was categorised by age group. Age, vaccine and non-vaccine serotype specific IR were calculated and pre-PCV7 vaccine (2007-2008), pre-PCV-13 vaccine (2009-2010) and post-vaccine (2015-2016) epidemiological years (July-June) were compared. Data analysis was performed using IBM SPSS Statistics for Windows, Version 20.0 (Armonk, NY: IBM Corporation) and software for calculating incidence rate ratios (IRR) and $95 \%$ confidence intervals (CI) (https://www.medcalc.org). Two-tailed $P$ value $\leqslant 0.05$ was considered statistically significant.

\section{RESULTS}

Over the 9 epidemiological years, $86 \%$ of all notified cases had typing data available (mean $86 \%$, range $79-92 \%)$. The annual proportion of typed isolate was $84,82,79,88,92,87,83,90$ and $90 \%$ for each epidemiological year between 2007-2008 and 2015-2016. This consisted of a total of 1387 isolates serotyped by the reference laboratory from samples taken from adults $\geqslant 65$ years of age from July 2007 to June 2016. Ninety-nine per cent of the isolates were from blood cultures.

Older adults aged between 75 and 84 years of age and those aged $\geqslant 85$ years had a consistently much higher IR than those aged between 65 and 74 years of age (Fig. 1). Although there was no statistically significant change in IR from 2007-2008 to 20152016, there was also a notable decline in IR in adults $\geqslant 85$ years of age in 2008-2009 and 2010 2011, which coincided with the introduction of PCV7 (September 2008) and PCV13 (December 2010). Overall there was much greater variability in the annual IR within this age groups than those aged between 65 and 74 years or those aged between 75 and 84 years of age. However, there was no statistically significant change over the 9 years in the total IPD IR in any of the three age categories and no significant change in the overall IR of all adults $\geqslant 65$ years of age which went from 28.66/100 000 in 2007-2008 to $28 \cdot 88 / 100000$ in $2015-2016$ (IRR $1 \cdot 01,95 \%$ CI $0 \cdot 80$ $1 \cdot 27, P=0.9477-$ as indicated in Table 1$)$.

Figure 2 displays the changes in vaccine-associated serotypes responsible for IPD from 2007-2008 to $2015-2016$ from all adults $\geqslant 65$ years of age (annual IR and breakdown for each serotype are also listed in Supplementary Table 2). The incidence of PCV7 serotypes fell rapidly after the introduction of PCV and declined consistently each year, including after PCV7 was replaced with PCV13. However the incidence of PCV13-7 serotypes remained relatively 


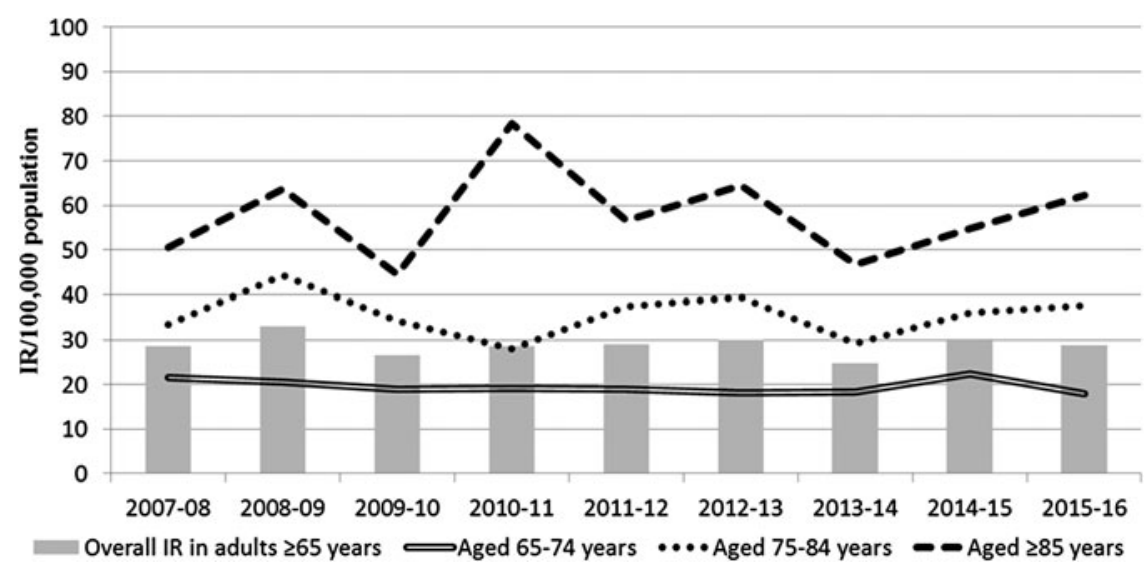

Fig. 1. The IPD incidence rate (IR) per 100000 population in Ireland by age group, from 2007-08 to $2015-16$.

unchanged, consistently staying $>6 / 100000$ and peaking in 2011-2012 (IR 10·16), despite the introduction of the PCV13 vaccine in December 2010. Moreover, the incidence of PPV23-PCV13 and non-vaccine types (NVTs) continued to increase. As indicated in Table 1, the incidence of PCV7 serotypes fell significantly (IRR $0 \cdot 05,95 \%$ CI $0 \cdot 01-0 \cdot 13, P<0 \cdot 0001$ ), however, there was no change in the incidence of PCV13-7 serotypes during this surveillance period (IRR $0 \cdot 90,95 \%$ CI $0 \cdot 54-1 \cdot 49, P=0 \cdot 6580$ ) nor in comparison with the pre-PCV13 year 2009-2010 (IRR $0.92,95 \%$ CI $0.56-1.53, P=0.7380$ ). However, there were significant increases in the incidence of PPV23-PCV13 (IRR $2 \cdot 17,95 \%$ CI $1 \cdot 40-3 \cdot 44, P=$ $0 \cdot 0002$ ) and NVTs (IRR $3 \cdot 43,95 \%$ CI $1 \cdot 81-7 \cdot 06, P$ $<0.0001$ ), this trend was evident in all three age groups (as displayed in Table 1).

The differences in serotypes associated IPD infections in 2007-2008 and 2015-2016 are listed in Supplementary Tables $1-3$. The results indicate that serotypes 3 (IRR 1·76, 95\% CI $0 \cdot 68-5 \cdot 11$ ) and 19A (IRR $1 \cdot 40,95 \%$ CI $0 \cdot 61-3 \cdot 42$ ) remain predominant and the number of cases has increased slightly in recent years in comparison with earlier years. However, it is also important to note that the incidence of PCV13-7 serotypes peaked in 2011-2012 (IR $10 \cdot 16 / 100000$ ), this may be also due to selective pressure following the introduction of $\mathrm{PCV} 7$ in September 2008, which resulted in a steady increase in PCV13-7 serotypes. Most of the PCV13-7 serotypes have subsequently fallen, such as $6 \mathrm{~A}$ and $7 \mathrm{~F}$, while others such as $19 \mathrm{~A}$ and 3 have remained predominant serotypes associated with invasive infections. Other serotypes that have also increased in recent years include PPV23-PCV13 serotypes 8, 10A,
$12 \mathrm{~F}, 17 \mathrm{~F}, 22 \mathrm{~F}, 33 \mathrm{~F}$ and the NVTs $15 \mathrm{~A}$ and $35 \mathrm{~F}$ (Supplementary Table 3).

As displayed in Table 2, a small subset of pneumococcal serotypes was associated with reduced susceptibility to penicillin (referred to as penicillin non-susceptible pneumococci, PNSP). A similar trend was observed for cefotaxime; an isolate nonsusceptible to penicillin was also more likely to be cefotaxime non-susceptible pneumococci (CNSP). The percentage of isolates that were identified as PNSP has fallen from a peak of $24 \%$ in $2008-2009$ to $13 \%$ in $2015-2016$. This was mainly due to a decline in PCV7 serotypes such as $6 \mathrm{~B}, 9 \mathrm{~V}, 14$ and $19 \mathrm{~F}$ that were also frequently associated with nonsusceptibility. However, the PCV13-7 serotype 19A has remained a predominant serotype associated with PNSP $(53 \%$ of serotype 19A isolates were PNSP in 2015-2016). Moreover, other serotypes not covered in any of the vaccines have emerged in recent years including 15A, 35B and 35F, with PNSP rates of 48,81 and $18 \%$ for the 9 -year period.

\section{DISCUSSION}

Retrospective analysis of serotypes distribution in IPD cases plays an important role in informing future vaccination strategies. It cannot be assumed that a decline in IPD incidence rates observed in some countries will always be mirrored elsewhere over time. There are some notable differences in our data compared with those from other national and international surveillance systems that are of interest. First, we did not see a consistent decline in PCV13-7 serotypes in adults $\geqslant 65$ years of age following the introduction of PCV13 to routine childhood vaccination in 2010. It was 
Table 1. Changes in the incidence of IPD in adults aged $\geqslant 65$ years of age, per age and vaccine-type

\begin{tabular}{|c|c|c|c|c|c|c|c|c|c|c|c|c|c|c|c|}
\hline & \multirow[b]{3}{*}{ Age and vaccine-type } & \multicolumn{6}{|c|}{ Epidemiological year } & \multicolumn{4}{|c|}{$\begin{array}{l}\text { Comparison of } 2007-2008 \text { with } 2015-2016 \\
\text { (pre-PCV7 era) }\end{array}$} & \multicolumn{4}{|c|}{$\begin{array}{l}\text { Comparison of } 2009-2010 \text { with } 2015- \\
2016 \text { (pre-PCV13 era) }\end{array}$} \\
\hline & & \multicolumn{2}{|c|}{ 2007-2008 } & \multicolumn{2}{|c|}{ 2009-2010 } & \multicolumn{2}{|c|}{ 2015-2016 } & \multirow[b]{2}{*}{$\%$ change* } & \multirow[b]{2}{*}{ IRR } & \multirow[b]{2}{*}{$95 \% \mathrm{CI}$} & \multirow[b]{2}{*}{$P$ value } & \multirow[b]{2}{*}{$\%$ change* } & \multirow[b]{2}{*}{ IRR } & \multirow{2}{*}{$\begin{array}{l}95 \% \mathrm{CI} \\
\text { Lower }\end{array}$} & \multirow[b]{2}{*}{$P$ value } \\
\hline & & IR & $n=$ & IR & $n=$ & IR & $n=$ & & & & & & & & \\
\hline \multirow[t]{5}{*}{ Adults aged $65-74$ years } & Total & $21 \cdot 63$ & 57 & $18 \cdot 85$ & 53 & $17 \cdot 90$ & 63 & +11 & $0 \cdot 83$ & $0 \cdot 57-1 \cdot 21$ & $0 \cdot 2997$ & +19 & $0 \cdot 95$ & $0 \cdot 65-1 \cdot 40$ & $0 \cdot 7814$ \\
\hline & PCV7 & $9 \cdot 11$ & 24 & $6 \cdot 76$ & 19 & $0 \cdot 00$ & 0 & -100 & $0 \cdot 00$ & $0 \cdot 00-0 \cdot 12$ & $<0 \cdot 0001$ & -100 & $0 \cdot 00$ & $0 \cdot 00-0 \cdot 17$ & $<0 \cdot 0001$ \\
\hline & PCV13-7 & $5 \cdot 69$ & 15 & $3 \cdot 56$ & 10 & $3 \cdot 12$ & 11 & -27 & $0 \cdot 55$ & $0 \cdot 23-1 \cdot 28$ & $0 \cdot 1253$ & +10 & $0 \cdot 88$ & $0 \cdot 34-2 \cdot 31$ & $0 \cdot 7672$ \\
\hline & PPV23-PCV13 & $4 \cdot 17$ & 11 & $6 \cdot 76$ & 19 & $10 \cdot 51$ & 37 & +236 & $2 \cdot 52$ & $1 \cdot 26-5 \cdot 47$ & $0 \cdot 0053$ & +95 & $1 \cdot 56$ & $0 \cdot 87-2 \cdot 86$ & $0 \cdot 1144$ \\
\hline & NVT & $2 \cdot 66$ & 7 & $1 \cdot 78$ & 5 & $4 \cdot 26$ & 15 & +114 & $1 \cdot 60$ & $0 \cdot 62-4 \cdot 65$ & $0 \cdot 2972$ & +200 & $2 \cdot 40$ & $0 \cdot 83-8 \cdot 43$ & $0 \cdot 0806$ \\
\hline \multirow[t]{5}{*}{ Adults aged $75-84$ years } & Total & $33 \cdot 53$ & 53 & $34 \cdot 20$ & 56 & $37 \cdot 76$ & 71 & +34 & $1 \cdot 13$ & $0 \cdot 78-1 \cdot 64$ & $0 \cdot 5122$ & +27 & $1 \cdot 10$ & $0 \cdot 77-1 \cdot 6$ & $0 \cdot 5799$ \\
\hline & PCV7 & $16 \cdot 45$ & 26 & $12 \cdot 22$ & 20 & $0 \cdot 53$ & 1 & 96 & $\mathbf{0 \cdot 0 3}$ & $0 \cdot 00-0 \cdot 20$ & $<0 \cdot 0001$ & -95 & $0 \cdot 04$ & $\mathbf{0} \cdot 00-0 \cdot 27$ & $<0 \cdot 0001$ \\
\hline & PCV13-7 & $8 \cdot 22$ & 13 & $11 \cdot 61$ & 19 & $9 \cdot 04$ & 17 & +31 & $1 \cdot 10$ & $0 \cdot 50-2 \cdot 46$ & 0.7969 & -11 & $0 \cdot 78$ & $0 \cdot 38-1 \cdot 58$ & $0 \cdot 4534$ \\
\hline & PPV23-PCV13 & $8 \cdot 22$ & 13 & $6 \cdot 11$ & 10 & $15 \cdot 95$ & 30 & +131 & 1.94 & $0 \cdot 98-4 \cdot 05$ & $0 \cdot 0421$ & +200 & $2 \cdot 61$ & $1 \cdot 24-5 \cdot 99$ & $0 \cdot 0063$ \\
\hline & NVT & $0 \cdot 63$ & 1 & $4 \cdot 28$ & 7 & $12 \cdot 23$ & 23 & +2200 & $19 \cdot 34$ & $3 \cdot 14-796 \cdot 54$ & $<0 \cdot 0001$ & +229 & $2 \cdot 86$ & $1 \cdot 19-7 \cdot 89$ & $0 \cdot 0108$ \\
\hline \multirow[t]{5}{*}{ Adults aged $\geqslant 85$ years } & Total & $50 \cdot 62$ & 25 & $44 \cdot 47$ & 24 & $62 \cdot 15$ & 41 & +64 & $1 \cdot 23$ & $0 \cdot 73-2 \cdot 11$ & $0 \cdot 4183$ & +71 & $1 \cdot 40$ & $0 \cdot 82-2 \cdot 42$ & $0 \cdot 1907$ \\
\hline & PCV7 & $24 \cdot 3$ & 12 & $20 \cdot 38$ & 11 & $4 \cdot 55$ & 3 & -75 & $0 \cdot 19$ & $0 \cdot 03-0 \cdot 69$ & $0 \cdot 0036$ & -73 & $0 \cdot 22$ & $0 \cdot 04-0 \cdot 84$ & $0 \cdot 0116$ \\
\hline & PCV13-7 & $8 \cdot 10$ & 4 & $7 \cdot 41$ & 4 & $13 \cdot 64$ & 9 & +125 & 1.68 & $0 \cdot 47-7 \cdot 48$ & $0 \cdot 3803$ & +125 & $1 \cdot 84$ & $0 \cdot 51-8 \cdot 18$ & $0 \cdot 3024$ \\
\hline & PPV23-PCV13 & $10 \cdot 12$ & 5 & $9 \cdot 26$ & 5 & $21 \cdot 22$ & 14 & +180 & $2 \cdot 10$ & $0 \cdot 71-7 \cdot 44$ & $0 \cdot 1462$ & +180 & $2 \cdot 29$ & $0 \cdot 78-8 \cdot 13$ & $0 \cdot 1017$ \\
\hline & NVT & $8 \cdot 10$ & 4 & $7 \cdot 41$ & 4 & $22 \cdot 74$ & 15 & +275 & $2 \cdot 81$ & $0 \cdot 89-11 \cdot 62$ & $0 \cdot 0553$ & +275 & 3.07 & $0 \cdot 98-12 \cdot 7$ & $0 \cdot 0359$ \\
\hline \multirow[t]{5}{*}{ Adults $\geqslant 65$ years } & Total & $28 \cdot 66$ & 135 & $26 \cdot 66$ & 133 & $28 \cdot 88$ & 175 & +30 & $1 \cdot 01$ & $0 \cdot 80-1 \cdot 27$ & $0 \cdot 9477$ & +32 & $1 \cdot 08$ & $0 \cdot 86-1 \cdot 37$ & $0 \cdot 4869$ \\
\hline & PCV7 & $13 \cdot 16$ & 62 & $10 \cdot 02$ & 50 & 0.66 & 4 & -94 & 0.05 & $0 \cdot 01-0 \cdot 13$ & $<0 \cdot 0001$ & -92 & $0 \cdot 07$ & $0 \cdot 02-0 \cdot 18$ & $<0 \cdot 0001$ \\
\hline & PCV13-7 & $6 \cdot 79$ & 32 & $6 \cdot 61$ & 33 & $6 \cdot 11$ & 37 & +16 & $0 \cdot 90$ & $0 \cdot 54-1 \cdot 49$ & $0 \cdot 6580$ & +12 & $0 \cdot 92$ & $0 \cdot 56-1 \cdot 53$ & $0 \cdot 7380$ \\
\hline & PPV23-PCV13 & $6 \cdot 16$ & 29 & $6 \cdot 81$ & 34 & $13 \cdot 37$ & 81 & 179 & $2 \cdot 17$ & $1 \cdot 4-3 \cdot 44$ & $0 \cdot 0002$ & +138 & 1.96 & $1 \cdot 30-3 \cdot 02$ & $0 \cdot 0008$ \\
\hline & NVT & $2 \cdot 55$ & 12 & $3 \cdot 21$ & 16 & $8 \cdot 75$ & 53 & +342 & $3 \cdot 43$ & $1 \cdot 81-7 \cdot 06$ & $<0 \cdot 0001$ & +231 & $2 \cdot 73$ & $1 \cdot 54-5 \cdot 11$ & $0 \cdot 0020$ \\
\hline
\end{tabular}

*\% change denotes the percentage change in the number of isolates typed in 2007-2008 (pre-PCV7) and 2009-2010 (pre-PCV13) in comparison with the number of isolates typed in 2015-2016

PCV7 denotes the serotypes in the 7-valent conjugate vaccine.

PCV13-7 denotes the serotypes in the 13-valent conjugate vaccine not included in PCV7.

PPV23-PCV13 denotes the serotypes in the 23-valent polysaccharide vaccine not included in PCV13.

Non-vaccine types (NVT) denote all other serotypes not included in either the PCV7/13 or PPV23 vaccines and non-typable isolates.

IR denotes the incidence rate of typed isolates per 100000 population per epidemiological year.

$n$, number of isolates typed per epidemiological year.

Incidence Rate Ratio (IRR) denotes the ratio between the incidence rate of typed isolates in the epidemiological year 2015-2016 in comparison with the pre-PCV7-vaccine epidemiological year 2007-2008 and pre-PCV13 vaccine year 2009-2010.

$95 \%$ CI denotes the $95 \%$ confidence interval of the IRR.

$P$ value of $<0.05$ denotes a significant difference between two incidence rates (highlighted in bold text). 


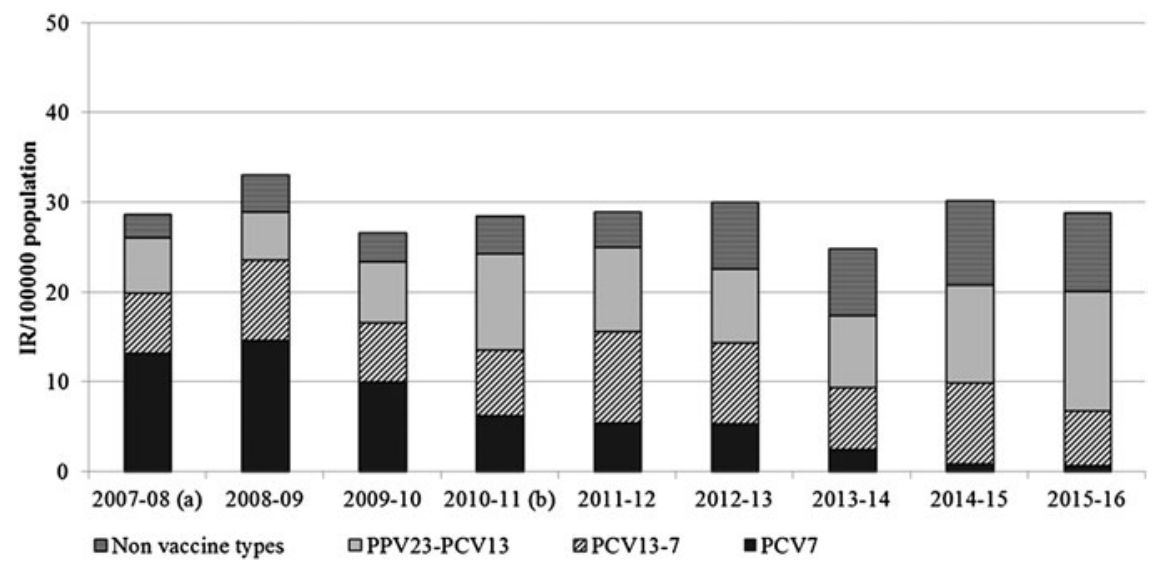

Fig. 2. The IPD incidence rate (IR) in adults aged $\geqslant 65$ years of age in Ireland, per serotype group based on epidemiological years (July 2007-June 2016). PCV7 denotes the serotypes in the 7-valent conjugate vaccine. PCV13-7 denotes the serotypes in the 13-valent conjugate vaccine not included in PCV7. PPV23-PCV13 denotes the serotypes in the 23-valent polysaccharide vaccine not included in PCV13. Non-vaccine types (NV1) denote all other serotypes not included

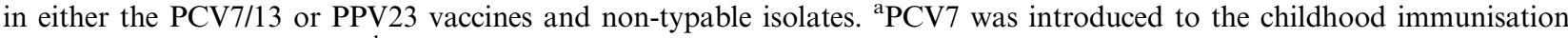
schedule in September 2008. ${ }^{\text {bCV}} 13$ was introduced to the childhood immunisation schedule in December 2010.

expected that PCV13 might provide indirect herdprotection to adults, similar to the preceding PCV7 vaccine. This unanticipated finding was attributable to the persistence/increase in serotypes 3 and 19A, which remained predominant serotypes in this age-group in Ireland in over 5 years following PCV13 introduction. Waight et al. reported a significant decline in the PCV13-7 serotypes 4 years after PCV13 introduction, with a notable decline in the incidence of serotypes 3, 7F and 19A by 2013-2014 (IRR: $0 \cdot 56,0 \cdot 44,0 \cdot 35$ respectively), which contributed to an overall fall of the IPD in adults $\geqslant 65$ years of age in the UK (IRR: 0.75) [6]. The Irish data display a similar decline in $7 \mathrm{~F}$ (IRR 0.49) but in contrast, there were increases in both serotype 3 (IRR 1.76) and 19A (IRR 1.40) by 2015-2016 in comparison with pre-PCV13 year 2009-2010, which led to no overall change in the incidence of IPD in adults $\geqslant 65$ years of age over the surveillance period (IRR $1 \cdot 01)$. These two serotypes were also the predominant serotypes in most other years (Supplementary Tables 1-3). Surveillance in the USA has also shown an overall decline in IPD in adults $\geqslant 65$ years of age from 2012-2013, which was driven by a decline in PCV13-7 serotypes (58\%), in particular serotypes $19 \mathrm{~A}$ and $7 \mathrm{~F}(58-72 \%)$ [9].

The difference in incidence of PCV-serotype infections may partly be due to differences in infant vaccine schedules. Both the UK and Ireland have a similar $2+1$ infant immunisation schedule with a catch-up campaign for children $<2$ years of age and have maintained a high vaccine uptake rate of $>90 \%[20$, $36]$. However, there were minor differences in the months of immunisation $(2,4,12$ months in UK and 2, 6, 12 months in Ireland) and introduction dates (PCV7 was introduced to the immunisation schedule in the UK in September 2006 and September 2008 in Ireland). Both countries introduced PCV13 in 2010, without a catch-up campaign $[6,19]$. As of 2015, Ireland switched to administering a final dose of PCV13 at 13 months to accommodate vaccination with the meningitis type $B$ infection at 12 months. The USA implemented a $3+1$ infant immunisation schedule (2, 4, 6 and 12-15 months) for PCV7 in 2000 and PCV13 in 2010 [9]. These differences may have also impacted on serotypes associated with colonisation within the paediatric population and lead to less herd-protection. However, despite differences in schedules, the incidence of PCV13-7 serotypes in the paediatric population fell significantly in Ireland following the introduction of PCV13 and is similar to what has been reported in other countries $[6,8,9]$.

Moreover, the USA has observed a smaller increase in non-PCV serotypes by $2012(7 \%)$ compared with our data where we observed an increase in IR of 13.4/100 000 for PPV23-PCV13 and NVT from 2007-2008 to 2015-2016, data derived from calculating difference in IR for PPV23-PCV + NVT, as listed in Table 1. By 2012-2013 we had already observed a doubling increase in non-PCV serotypes (Figure 2), which continued to increase for both PPV23-PCV13 (IRR 2.17) and NVTs (IRR 3.43) (Table 1). The 


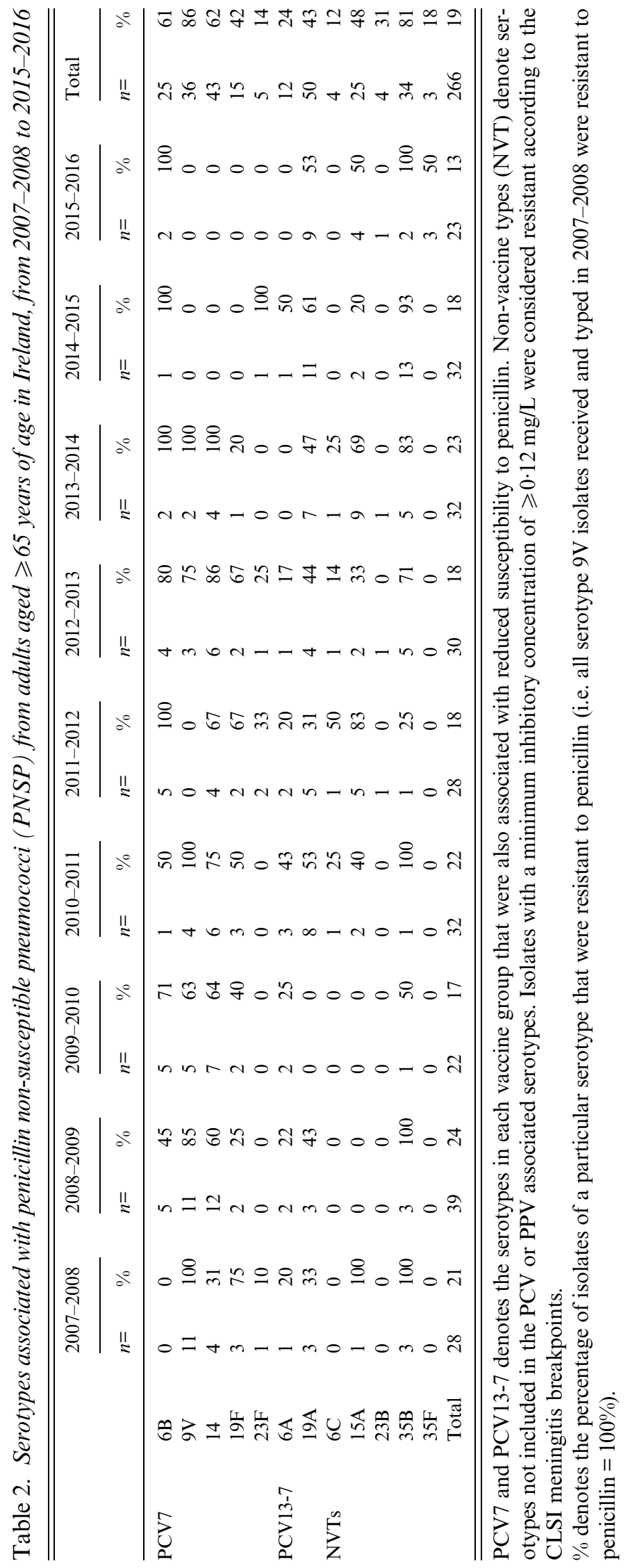

UK, also reported a more modest increase in non-PCV serotype infections in adults $\geqslant 65$ years (IRR 1.25) in comparison with our data. The predominant non-PCV vaccine serotypes associated with IPD in our data include serotypes $8,12 \mathrm{~F}, 22 \mathrm{~F}, 33 \mathrm{~F}$, which are all included in the PPV23 vaccine, and two serotypes that are not included in the current PPV23 or PCV13, serotypes 15A and 35B. These two serotypes are also frequently associated with resistance.

The effectiveness of PPV23 in the elderly is uncertain as this is almost exclusively based on observations studies that are prone to confounding variables, particularly its use amongst healthy individuals, which is impossible to fully correct for. Given that the incidence of PCV13-7 serotypes has remained high in Ireland, coupled with increasing PPV23-PCV13 serotypes; it is likely that introducing one dose of PCV13 in addition to PPV23 for at-risk adults or those aged $\geqslant 65$ years of age could reduce the burden of IPD in adults. The USA has already updated the vaccination schedule to include one dose of PCV13 for adults $\geqslant 65$ years of age in order to further reduce the burden of IPD, despite a decline in PCV13 from the childhood vaccination schedule [15]. However, the vaccination uptake rate of PPV23 would need to be improved from $27-36 \%$ in Ireland to reduce the burden of IPD [23]. Bedford et al. found that approximately $80 \%$ of older persons in Ireland would consider availing of the pneumococcal vaccine from their doctor if requested [37]. Alternatively, recognising the opportunity to vaccinate individuals while in medical facilities may improve uptake rates [38]. It is likely that other co-morbidities contribute to the high rate of IPD in Ireland. A recent EU report found that Ireland had a higher mortality rate than the EU average for a number of respiratory related diseases including asthma $(1 \cdot 7 \mathrm{vs} .1 \cdot 3 / 100000)$, pneumonia (Ireland: $40 \cdot 3 \mathrm{vs}$. EU average: $27 \cdot 5 / 100000$ ), chronic respiratory illness $(63 \cdot 7$ vs. $38 \cdot 3 / 100000)$, and other respiratory diseases $(62 \cdot 0$ vs. 37.0/100 000) [39]. Factors such as poor diet, smoking and poor awareness of lung disease may also contribute to increased risk of infection in the adult population [40]. Interestingly, increased interaction with children, such as the provision of childcare, may also contribute to carriage of pneumococcal serotypes in the older adult population [41].

Vaccine replacement serotypes are emerging in both adult and paediatric cohorts $[6,8,9,11,16,18]$ and the proportion of serotypes varies in different regions with no one or two 'leading' serotypes emerging in multiple sites, in comparison with the previous 
emergence of 7F and 19A [18]. As a result, higher valency vaccines to include a number of emerging serotypes or new vaccine targets that move away from serotype specific differences may be needed to address this challenge [42].

There are some limitations associated with the use of surveillance studies for comparison of incidence rates over several years. First, diagnostic and referral policies vary over time and between referring hospitals. However, the results of a recent user survey of clinical laboratories in Ireland have confirmed that despite the introduction of new molecular technology, the use of routine culture techniques remains the 'gold standard' for the detection of $S$. pneumoniae from samples from sterile sites. Moreover, the number of case notifications and referred isolates is monitored on a quarterly basis and notable changes are discussed directly with the hospitals. It is also important to note that the statutory obligation of notifying all confirmed IPD cases in Ireland has been in place since 2004 and has not changed since the introduction of typing services in 2007. Matching of individual serotype results obtained from samples sent to the IPRL with notification data has also allowed us to detect any discrepancies between the numbers notified and those referred for typing. Second, an increase in the percentage of cases serotyped could lead to biased estimates of the vaccination-effect and the degree of serotype replacement. The number of isolates collected over the 9 years is relatively small (total $n=1387$ ), which may result in stochastic changes. However, this limitation is due to the small population in Ireland in comparison with bigger countries with access to larger culture collections. In order to correct this our data are also stratified using population statistics in order to allow comparison with countries with larger population and the use of $95 \%$ confidence intervals for all incidence rate comparisons. Moreover, as a result of matching notification data with typing results, we calculate that $86 \%$ (range $79-92 \%$ ) of all IPD cases reported from adults $\geqslant 65$ years of age from July 2007 to June 2016 were typed, with no discernible time trend. This also provides confidence that the trends observed are an accurate representation of trends of IPD in Ireland during this period. Consequently, we are confident that the trends reported in this paper are a reliable reflection of serotype distribution associated with IPD in Ireland. Finally, while the vaccination uptake rates are monitored as part of the infant immunisation scheme, there is currently no similar scheme for routinely monitoring pneumococcal vaccination uptake in adults, aside from intermittent surveys taken in 2005, 2010 and 2013, which indicate that only between 27 and $36 \%$ of adults $\geqslant 65$ years of age had received the vaccine [23]. This may be considered a limitation of this study as it is difficult to accurately estimate the direct effects of vaccination without precise monitoring of vaccination uptake in adults. Our groups are currently involved in a European study assessing the vaccination uptake rate in adults in more detail and we are hoping this will also allow us to assess the numbers needed to vaccinate in order to provide protection for this aging population. It would also be of great interest to further evaluate the figures surrounding the switch from PCV7 to PCV13 in the paediatric population in more detail; however, aside from overall uptake rates this data are not available to us at this time [19].

In conclusion, the number of infections caused by vaccine serotypes has fallen in Ireland but most of this is from serotypes covered in PCV7, which provided herd-protection for adults promptly after the vaccine was introduced. However, the number of IPD infections associated with PCV13-7 serotypes has remained relatively unchanged despite the introduction of PCV13 over 5 years ago and those covered in PPV23-PCV13 and NVTs have increased significantly. As a result, the administration of both PCV13 and PPV23 in adults may reduce the incidence of IPD infections and should be considered.

\section{SUPPLEMENTARY MATERIAL}

The supplementary material for this article can be found at https://doi.org/10.1017/S0950268817001194.

\section{ACKNOWLEDGEMENTS}

We wish to thank all the laboratory scientists and consultant microbiologists in Irish hospitals for forwarding pneumococcal isolates and all the staff in the Departments of Public Health involved in the surveillance of pneumococcal disease in Ireland.

This work is partly funded by the Health Protection Surveillance Centre (Ireland). Additional funding has been provided through an unrestricted research grant from Pfizer (Ireland). The funders had no role in the collection, analysis, interpretation of data or in the writing of and decision to submit the article for publication. 


\section{DECLARATION OF INTEREST}

HH has recently received research funds from Astellas and Pfizer, and has received lecture and other fees from Cepheid and Astellas. MCo, IV and MMcE have received funding support from Pfizer.

\section{AUTHORS CONTRIBUTIONS}

$\mathrm{HH}, \mathrm{MCa}, \mathrm{SC}$ and DO'F were involved in the establishment of pneumococcal serotyping service. IV $\mathrm{MMcE}$ and MCo have carried out the serotyping and antimicrobial susceptibility testing and JM, MF, SM, SC, DO'F and RC have developed and led national surveillance of IPD. MCo, HH, IV and RC have drafted the manuscript which has been reviewed and agreed by all.

\section{REFERENCES}

1. World Health Organisation. Priority medicines for Europe and the world 'a public health approach to innovation' (http://www.who.int/medicines/areas/ priority_medicines/BP6_22Pneumo.pdf). May 2013.

2. Richter SS, et al. Pneumococcal serotypes before and after introduction of conjugate vaccines, United States, 1999-2011. Emerging Infectious Diseases 2013; 7: 1074-1083.

3. Whitney CG. Changing epidemiology of pneumococcal disease in the era of conjugate vaccines. Current Epidemiology Reports 2016; 3: 125-135.

4. Weinberger DM, Malley R, Lipsitch M. Serotype replacement in disease following pneumococcal vaccination: a discussion of the evidence. Lancet 2011; 378: 1962-1973.

5. Geno KA, et al. Pneumococcal capsules and their types: past, present, and future. Clinical Microbiology Reviews 2015; 28: 871-899.

6. Waight PA, et al. Effect of the 13-valent pneumococcal conjugate vaccine on invasive pneumococcal disease in England and Wales 4 years after its introduction: an observational cohort study. Lancet Infectious Diseases 2015; 15: 535-543.

7. van der Linden M, et al. Four years of universal pneumococcal conjugate infant vaccination in Germany: impact on incidence of invasive pneumococcal disease and serotype distribution in children. Vaccine 2014; 30: $5880-5885$.

8. van der Linden M, et al. Effects of infant pneumococcal conjugate vaccination on serotype distribution in invasive pneumococcal disease among children and adults in Germany. PLoS ONE 2015; 10: e0131494.

9. Moore MR, et al. Effect of use of 13-valent pneumococcal conjugate vaccine in children on invasive pneumococcal disease in children and adults in the USA: analysis of multisite, population-based surveillance. Lancet Infectious Diseases 2015; 15: 301-309.
10. Regev-Yochay G, et al. Early impact of PCV7/PCV13 sequential introduction to the national pediatric immunization plan, on adult invasive pneumococcal disease: a nationwide surveillance study. Vaccine 2015; 33: 1135 1142.

11. Steens A, Vestrheim DF, de Blasio BF. Pneumococcal vaccination in older adults in the era of childhood vaccination: public health insights from a Norwegian statistical prediction study. Epidemics 2015; 11: 24-31.

12. Harboe ZB, et al. Impact of 13-valent pneumococcal conjugate vaccination in invasive pneumococcal disease incidence and mortality. Clinical Infectious Diseases 2014; 59: 1066-1073.

13. European Centre for Disease Control. Recommended immunisations for pneumococcal disease. 2016 (http:// vaccine-schedule.ecdc.europa.eu/Pages/Scheduler.aspx). Accessed 1 September 2016.

14. Castiglia P. Recommendations for pneumococcal immunization outside routine childhood immunization programs in western Europe. Advances in Therapy 2014; 31: 1011-1044.

15. Williams WW, et al. Surveillance of vaccination coverage among adult populations - United States, 2014. Morbidity and Mortality Weekly Report 2016; 1: $1-36$.

16. Hausdorff WP, Hanage WP. Interim results of an ecological experiment - conjugate vaccination against the pneumococcus and serotype replacement. Human Vaccines and Immunotherapeutics 2016; 12: 358-374.

17. Feikin DR, et al. Serotype-specific changes in invasive pneumococcal disease after pneumococcal conjugate vaccine introduction: a pooled analysis of multiple surveillance sites. PLoS Medicine 2013; 10: e1001517.

18. Tin Tin Htar M, Christopoulou D, Schmitt HJ. Pneumococcal serotype evolution in Western Europe. BMC Infectious Diseases 2015; 15: 1-10.

19. Health Protection Surveillance Centre. Immunisation uptake report for Ireland - A report by the Health Protection Surveillance Centre-2016 (http://www.hpsc. ie/A-Z/VaccinePreventable/Vaccination/Immunisation UptakeStatistics/Immunisationuptakestatisticsat12and24 monthsofage/AnnualReports/File,15662,en.pdf). Accessed 31 August 2016.

20. Health Protection Surveillance Centre. Quarterly Reports on Invasive Pneumococcal Disease. 2016 (https:// www.hpsc.ie/A-Z/VaccinePreventable/Pneumococcal Disease/Publications/QuarterlyReportsonInvasive PneumococcaDisease/File,15801,en.pdf). Accessed 04 August 2016.

21. Harboe ZB, et al. pediatric invasive pneumococcal disease caused by vaccine serotypes following the introduction of conjugate vaccination in Denmark. PLoS ONE 2013; 8: e51460.

22. National Immunisation Advisory Council. Chapter 3 Immunisation of Immunocompromised Persons. 2015 (http://www.hse.ie/eng/health/immunisation/hcpinfo/ guidelines/chapter3.pdf). Accessed 02 February 2016.

23. Giese $\mathbf{C}$, et al. Low vaccination coverage for seasonal influenza and pneumococcal disease among adults at-risk and health care workers in Ireland, 2013: the 
key role of GPs in recommending vaccination. Vaccine 2016; 34: 3657-3662.

24. Theidel U, Kuhlmann A, Braem A. Pneumococcal vaccination rates in adults in Germany. Deutsches Ärzteblatt International 2013; 110: 743-750.

25. Public Health England. Pneumococcal Polysaccharide Vaccine (PPV) coverage report, England, April 2015 to March 2016 (https://www.gov.uk/government/ uploads/system/uploads/attachment_data/file/540290/hpr 2416_ppv.pdf). Accessed 05 July 2016.

26. Joint Committee on Vaccination and Immunisation. Interim JCVI statement on adult pneumococcal vaccination in the UK - November 2015 (https://www.gov.uk/ government/uploads/system/uploads/attachment_data/ file/477966/JCVI_pnemococcal.pdf). Accessed 03 September 2016.

27. Bonten MJM, et al. Polysaccharide conjugate vaccine against pneumococcal pneumonia in adults. New England Journal of Medicine 2015; 372: 1114-1125.

28. Patterson S, et al. A post hoc assessment of duration of protection in CAPiTA (Community Acquired Pneumonia immunization Trial in Adults). Trials in Vaccinology 2016; 5: 92-96.

29. Andrews NJ, et al. Impact and effectiveness of 23-valent pneumococcal polysaccharide vaccine against invasive pneumococcal disease in the elderly in England and Wales. Vaccine 2012; 30: 6802-6808.

30. Jackson LA, et al. Immunogenicity and safety of a 13-valent pneumococcal conjugate vaccine compared to a 23 -valent pneumococcal polysaccharide vaccine in pneumococcal vaccine-naive adults. Vaccine 2013; 31: 3577-3584.

31. Jackson LA, et al. Immunogenicity and safety of a 13 -valent pneumococcal conjugate vaccine in adults 70 years of age and older previously vaccinated with 23-valent pneumococcal polysaccharide vaccine. Vaccine 2013; 31: 3585-3593.

32. Tomczyk S, et al. Use of 13-valent pneumococcal conjugate vaccine and 23-valent pneumococcal polysaccharide vaccine among adults aged $\geqslant 65$ years: recommendations of the Advisory Committee on Immunization Practices (ACIP). Morbidity and Mortality Weekly Report 2014; 37: 822-825.

33. European Commission. Amending Decision 2002/253/ EC laying down case definitions for reporting communicable diseases to the Community network under
Decision No 2119/98/EC of the European Parliament and of the Council. Section L159/72. Official Journal of the European Union. 2002 [June 2016]. Available from: http://ec.europa.eu/health/ph_threats/com/docs/ 1589_2008_en.pdf.

34. Vickers I, O'Flanagan D, Cafferkey M, Humphreys H. Multiplex PCR to determine Streptococcus pneumoniae serotypes causing otitis media in the Republic of Ireland with further characterisation of antimicrobial susceptibilities and genotypes. European Journal of Clinical Microbiology and Infectious Diseases 2011; 30: 447-453.

35. Wayne P. Performance standards for antimicrobial susceptibility testing; twenty-third information supplement. CLSI document. M100-S23. Clinical Laboratory Standards Institute 2013.

36. Public Health England. Quarterly vaccination coverage statistics for children aged up to five years in the UK (COVER programme): January to March 2015 [September 2016]; Available from: https://www.gov.uk/ government/uploads/system/uploads/attachment_data/ file/438439/hpr2215_cover.pdf.

37. Bedford D, et al. The acceptability of pneumococcal vaccine to older persons in Ireland. Irish Medical Journal 2000; 93: 48-49.

38. Kyaw MH, et al. Adults with invasive pneumococcal disease. American Journal of Preventive Medicine 2006 2015/11/27; 31: 286-292.

39. European Statistics- Europa. Respiratory diseases statistics.2016 (http://ec.europa.eu/eurostat/statistics-explained/ index.php/Respiratory_diseases_statistics). Accessed 27 March 2017.

40. Irish Thoratic Society. Lung disease in Ireland - prevalence and trends: implications for work force planning (http://www.irishthoracicsociety.com/images/uploads/ Lung \%20Disease \%20in\%20Ireland\%20December $\% 20$ 2013.pdf). Accessed 27 March 2017.

41. Tramuto F. et al. Pneumococcal colonization in the familial context and implications for anti-pneumococcal immunization in adults: results from the BINOCOLO project in Sicily. International Journal of Molecular Sciences 2017; 18: 105.

42. Moffitt K, Malley R. Rationale and prospects for novel pneumococcal vaccines. Human Vaccines and Immunotherapeutics 2016; 12: 383-392. 